\title{
Rapid Eye Movement Sleep Behavior Disorder and Neurodegenerative Diseases: An Update
}

\author{
Feng Zhang1,2,\#, Long Niu ${ }^{1,2, \#, ~ X i n y a o ~ L i u ~}{ }^{1,2}$, Yufei Liu ${ }^{1,2}$, Song Li ${ }^{1,2}$, Huan Yu ${ }^{3}$, Weidong Le ${ }^{1,2, *}$ \\ ${ }^{1}$ Center for Clinical Research on Neurological Diseases, the First Affiliated Hospital, Dalian Medical University, \\ Dalian, China. \\ ${ }^{2}$ Liaoning Provincial Key Laboratory for Research on the Pathogenic Mechanisms of Neurological Diseases, the \\ First Affiliated Hospital, Dalian Medical University, Dalian, China. \\ ${ }^{3}$ Sleep and Wake Disorders Center and Department of Neurology, Huashan Hospital, Fudan University, Shanghai, \\ China.
}

[Received February 19, 2019; Revised March 18, 2019; Accepted March 20, 2019]

\begin{abstract}
Rapid eye movement sleep behavior disorder (RBD) is a sleep behavior disorder characterized by abnormal behaviors and loss of muscle atonia during rapid eye movement (REM) sleep. RBD is generally considered to be associated with synucleinopathies, such as Parkinson's disease (PD), dementia with Lewy bodies (DLB), and multiple system atrophy (MSA), and usually precedes years before the first symptom of these diseases. It is believed that RBD predicts the neurodegeneration in synucleinopathy. However, increasing evidences have shown that RBD is also found in non-synucleinopathy neurodegenerative diseases, including Alzheimer's disease (AD), Huntington's disease (HD), amyotrophic lateral sclerosis (ALS), etc. Sleep disturbance such as RBD may be an early sign of neurodegeneration in these diseases, and also serve as an assessment of cognitive impairments. In this review, we updated the clinical characteristics, diagnosis, and possible mechanisms of RBD in neurogenerative diseases. A better understanding of RBD in these neurogenerative diseases will provide biomarkers and novel therapeutics for the early diagnosis and treatment of the diseases.
\end{abstract}

Key words: rapid eye movement sleep behavior disorder, synucleinopathy, dementia, neurodegeneration

Rapid eye movement sleep behavior disorder (RBD) is a parasomnia characterized by abnormal behaviors and loss of muscle atonia, such as vocalizations, jerks and motor behaviors during rapid eye movement (REM) sleep, often related to REM-related dream content [1]. The prevalence of RBD is estimated to be $0.5-2 \%$, but larger populationbased studies of probable dream enactment symptoms suggest RBD is likely more frequent $(5-13 \%)$ in older community-dwelling adults [2]. The incidence of RBD is approximately equal in men and women under the age of 50 , but it appears to be more common in men than women in older adults [3]. Maybe it is because women have less violent dream enactment behaviors (DEBs) [4]. RBD can be categorized into idiopathic or symptomatic forms [3]. Idiopathic RBD (iRBD) often occurs in the absence of ongoing neurological conditions, whereas symptomatic RBD is related to identifiable underlying etiologies [5], such as $\alpha$-synuclein ( $\alpha$-syn) pathology and other forms of neurodegeneration, pontine lesions, and toxic effects from medications. Some evidence suggests that both idiopathic and symptomatic forms of RBD are associated with neurodegenerative diseases, especially $\alpha$-syn related

*Correspondence should be addressed to: Dr. Weidong Le, the First Affiliated Hospital, Dalian Medical University, Dalian 116021, China Email: wdle@sibs.ac.cn. \#These authors contributed equally to this work.

Copyright: ( 2019 Zhang F et al. This is an open-access article distributed under the terms of the Creative Commons Attribution License, which permits unrestricted use, distribution, and reproduction in any medium, provided the original author and source are credited. 
diseases [4]. iRBD is considered to be an important precursor of synucleinopathies such as Parkinson's disease (PD), dementia with Lewy bodies (DLB) and multiple system atrophy (MSA) [6, 7]. In fact, up to $82 \%$ of older men diagnosed with RBD develop parkinsonism or dementia [8]. Almost half of the PD patients, at least $88 \%$ of the MSA patients, and about $80 \%$ of the DLB patients have RBD [3]. Study has reported that up to $98 \%$ of individuals with polysomnography (PSG)-confirmed RBD have synucleinopathy [9]. Symptomatic RBD may also be associated with narcolepsy, Guillain Barre syndrome, limbic encephalitis and Morvan's syndrome [10]. RBD is recently recognized as part of the clinical spectrum of IgLON5-anti-associated sleep disorder [11]. Besides $\alpha$-syn diseases, RBD has been reported in nonsynucleinopathies, such as Alzheimer's disease (AD) [12], progressive supranuclear palsy (PSP) [13], Huntington's disease (HD) [14], and amyotrophic lateral sclerosis (ALS) [15].

\section{Clinical features and diagnosis of RBD}

RBD can cause sleep disruption, excessive motor activity ranging from simple limb twitches to violent, complex movements that may cause injury to the patients and/or sleeping partners [5]. These behaviors are often referred to as DEBs because patients often recall dreams immediately after waking up following an episode, and these behaviors seem to be purposeful [16]. Patients with RBD often have violent dream content [17]. The behaviors can occur more towards the morning hours because that is when REM sleep is more frequent, and the behaviors can start as early as 90 minutes after the first REM sleep episode [16]. Recent studies have showed that Epworth sleep scale $>8$ means more rapid conversion to $\mathrm{PD}$ and dementia at the time of iRBD diagnosis, and in some iRBD patients, cognitive impairment may reflect early stages of neurodegenerative diseases [18]. And if the patients with iRBD have hyposmia and constipation, orthostatic hypotension, and gait abnormalities, suggesting possible underlying pathologies of synucleinopathy [2].

The $3^{\text {rd }}$ revision diagnostic criteria of International Classification of Sleep Disorders Criteria for REM sleep behavior disorders consists (1) repeated sleep-related vocalizations and/or complex motor behaviors; (2) these behaviors are documented by polysomnography to occur during REM sleep, or based on the clinical history of dream enactment, are presumed to occur during REM sleep; (3) polysomnographic demonstrates REM sleep without atonia (RSWA); (4) the sleep disturbance is not better explained by another sleep disorder, mental disorder, medication, or substance use. [19].
Diagnosis of RBD requires either a clinical history of DEBs or REM sleep-related behaviors recorded by PSG, along with RSWA [2]. The witness of dream enactment by bed partner is the key to diagnose RBD [20]. RSWA is the electrophysiological hallmark of RBD, and also a key diagnostic feature of RBD [10, 21]. Some questionnaires can also be used in the diagnosis of RBD [22], such as RBD Screening Questionnaire (RBDSQ), the REM Sleep Behavior Questionnaires-Hong-Kong (RBD-HK), the Mayo Sleep Questionnaire (MSQ) and the Innsbruck RBD Inventory [2]. RBD needs to be distinguished from other similar diseases, such as non-REM parasomnias (sleepwalking, sleep terrors), periodic limb movement disorder, severe obstructive sleep apnea, and dissociative disorders, therefore PSG is very important for the diagnosis of RBD [10, 21]. Neuroimaging techniques might be helpful in detecting structural changes in RBD patients' brains. For example, diffusion tensor imaging (DTI), a widely used magnetic resonance imaging (MRI) technique, has been reported to detect white matter abnormalities in patients with iRBD [23]. White matter changes have been found in the brainstem where REM is modulated, and other brain areas, such as the right substantia nigra, the olfactory region, the left temporal lobe, the fornix, the internal capsule, the corona radiata, and the right visual stream in the patients with iRBD [23, 24]. However, other DTI studies have revealed slight or moderate changes in white matter of iRBD patients [25, 26]. A study of 3.0-T MRI has showed the loss of nigral hyperintensity in 11 iRBD patients, in consistent with a significant lower ${ }^{123} \mathrm{I}-\mathrm{N}$-3-fluoropropyl-2 $\beta$-carbomethoxy -3 $\beta$-4-iodophenyl tropane (a visualized tracer of striatal pre-synaptic dopamine transporter) uptake ratios [27]. Moreover, in a follow-up study of 5 iRBD patients with nigral hyperintensity loss, all patients have developed symptoms of parkinsonism or dementia 18 months after neuroimaging, implying that nigral hyperintensity loss at 3.0-T susceptibility-weighted imaging might be a marker for synucleinopathy risk in iRBD [27]. Overall, definite diagnosis of RBD is based on PSG confirmation. For identification or screening in large populations, single screening questions could be used followed by specific RBD rating scales and a more detailed sleep interview [28]. Other clinical symptoms such as olfactory loss, autonomic dysfunction, cognitive impairment, and examinations such as functional MRI, transcranial sonography, and peripheral nerve tissue biopsies may be helpful for mornitoring the outcome of RBD [28].

\section{Pathogenic mechanisms of RBD}

RBD may be triggered by neurodegenerative diseases or associated with antidepressant treatments, alcohol, and drug withdrawal [4]. But the mechanisms underlying the 
pathogenesis of RBD are still not clear. In rodents, the core of the REM-generating circuit includes $\gamma$ aminobutyric acid (GABA)-ergic neurons located in the lateral hypothalamus, dorsal paragigantocellular reticular nucleus, and ventrolateral periaqueductal grey (vlPAG) which inactivates REM-inhibiting monoaminergic neurons in the tuberomammillary nucleus, locus coeruleus (LC), and dorsal raphe and GABA-ergic neurons in the vIPAG to induce REM sleep [29]. The precoeruleus and sublaterodorsal nucleus (SLD) (equivalent to subcoeruleus nucleus (SubCD) in humans [30]) induce atonia during REM sleep in rodents [2]. SLD glutamatergic/GABA-ergic neurons stimulate inhibitory spinal interneurons, which in turn inhibit or hyperpolarize motor neurons to produce skeletal muscle atonia or stimulates glycinergic and GABA-ergic premotor neurons in the ventromedial medulla (VMM), including raphe magnus and lateral gigantocellular reticular nuclei (ventral gigantocellular, alpha gigantocellular and lateral paragigantocellular reticular nuclei), which result in inhibition of motor neurons and cause skeletal muscle atonia $[2,16,31]$. Blocking glycine and GABA receptors in the VMM via both pharmacological and genetic ways increases muscle twitches during REM sleep [29]. The cortical activation during REM sleep is restricted to a few limbic structures, such as retrosplenial cortex, medial entorhinal cortex, anterior cingulate cortex and the dentate gyrus, which produce dream and activate the motor cortex, leading to the activation of spinal motor neurons [31].

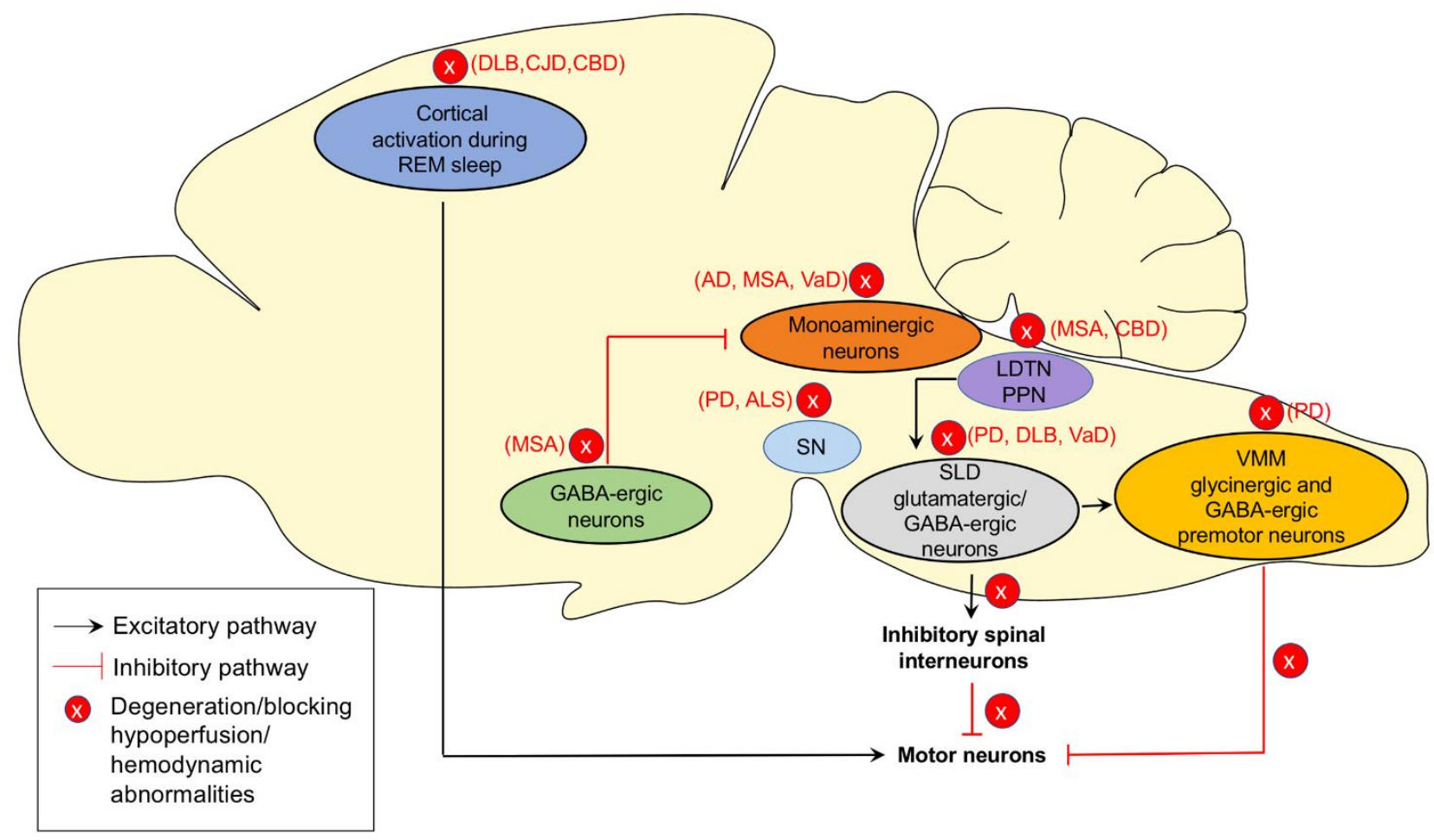

Figure 1. The potential mechanisms of RBD and related pathological pathways associated with neurodegenerative diseases. During rapid eye movement (REM) sleep, the $\gamma$-aminobutyric acid (GABA)-ergic neurons located in the lateral hypothalamus and other nuclei inactivate REM-inhibiting monoaminergic neurons in the tuberomammillary nucleus, locus coeruleus (LC), and dorsal raphe and GABA-ergic neurons in the ventrolateral periaqueductal grey (vlPAG) to induce REM sleep. Sublaterodorsal nucleus (SLD) glutamatergic/GABA-ergic neurons stimulate inhibitory spinal interneurons or glycinergic and GABA-ergic premotor neurons in the ventromedial medulla (VMM) resulting in skeletal muscle atonia. SLD neurons may be activated by the cholinergic laterodorsal tegmental nucleus and pedunculopontine tegmental nucleus (LDTN/PPN) neurons. The cortical activation during REM sleep leads to the activation of spinal motor neurons. Blocking glycine and GABA receptors or the degeneration of glycinergic and GABA-ergic neurons in the SLD and VMM removes the inhibition of spinal motor neurons and prevents the induction of muscle atonia. This could be the possible mechanism of RBD. In neurodegenerative diseases, pathological changes affecting the REM sleep regulating nuclei and circuits may contribute to the pathogenesis of RBD in these specific diseases. 
In healthy individuals, motor neuronal activation is inhibited by the SubCD and VMM, but in patients with $\mathrm{RBD}$, degeneration of glycinergic and GABA-ergic neurons in the SubCD and VMM removes the inhibition of spinal motor neurons and prevents the induction of muscle atonia [31]. Many other sites are also involved in REM sleep regulation, including pedunculopontine nucleus, laterodorsal tegmental nucleus (LDTN), thalamus, substantia nigra, basal forebrain, and frontal cortex [4]. The posterior hypothalamic hypocretin may have the effect of stabilizing REM-generating and REMinhibiting centers and networks [32]. The red nucleus, pedunculopontine nucleus, and LDTN may be the source of muscle twitches during REM sleep [32]. The circuits that regulate REM sleep in the caudal brainstem are the same structures where $\alpha$-syn pathology might begin [30]. Lesions in these structures are thought to eliminate atonia during REM sleep $[4,33]$. Autopsy evidence implies that
RBD patients have degeneration in brainstem nuclei that control REM sleep, with Lewy bodies and Lewy neurites in these areas [34]. Hypothesis suggests that lesions in the REM sleep circuits can lead to RBD-like motor behavior in both animals (cats, rats, mice) and humans [35]. Braak has used postmortem analysis of PD brains to propose a hypothesis that the $\alpha$-syn pathology progressively develops in a caudal to rostral fashion, this progression might be caused by cell-to-cell transmission between interconnected brain regions [36, 37]. The pathological changes begin from medulla and pons, which may be related to the development of initial RSWA and RBD in idiopathic RBD and eventually ascending to more rostral structures $[2,36]$. In most cases iRBD may not be separate disease entity from synucleinopathies, but rather an early manifestation of synaptophysin diseases.

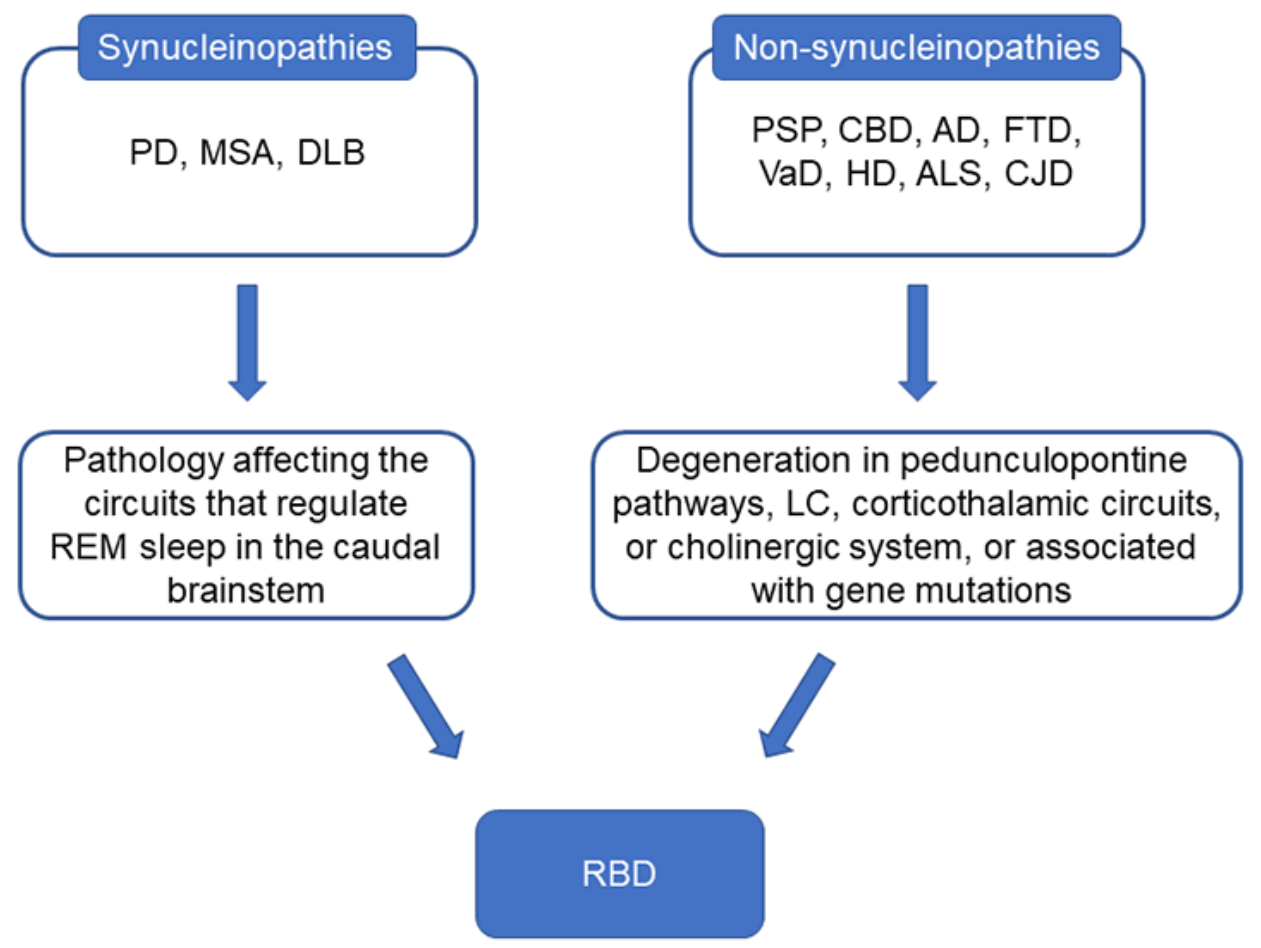

Figure 2. The possible mechanisms of RBD associated with synucleinopathies or nonsynucleinopathies. Lesions in caudal brainstem are thought to eliminate atonia during REM sleep and the brainstem is the same structure where $\alpha$-syn pathology might begin in synucleinopathies. In nonsynucleinopatheis, the pathological changes may affect pedunculopontine pathways, locus coeruleus (LC), corticothalamic circuits or cholinergic system and finally affect the REM sleep regulating systems to induce RBD.

In non-synucleinopathies, pathological changes affect pedunculopontine tegmentum or cholinergic system may induce RBD. For example, loss of cholinergic neurons in the pedunculopontine tegmentum in PSP and degenerative process in the nuclei of the brainstem and pedunculopontine pathways in corticobasal degeneration (CBD) may cause RBD in these patients [13, 38]. Moreover, in frontotemporal dementia (FTD) and ALS 
where $\alpha$-syn pathology is rarely found, RBD might exist alone without $\alpha$-syn. Degeneration of neurons in REMassociated pathways in the brainstem might contribute to RBD in ALS [15]. The plausible underlying mechanisms of RBD and related pathological pathways associated with neurodegenerative diseases are represented in Figure 1 and Figure 2.

So far, the research on the genetic factors of RBD is limited. Study shows that in patients with idiopathic RBD and $\mathrm{PD}$, there is a close association between RBD and the glucose encephalo-glucosidase ( $G B A)$ mutation [39]. PDassociated loci, scavenger receptor class $\mathrm{B}$ member 2 (SCARB2) rs6812193 and microtubule associated protein tau (MAPT) rs12185268 are also associated with RBD, while homozygous carriers of the ubiquitin specific peptidase 25 (USP25) rs2823357 single-nucleotide polymorphism (SNP) show a more rapid progression to synucleinopathy [40]. In RBD patients, PTEN-induced putative kinase 1 (PINK1) SNP rs45478900 carriers could have a higher risk of conversion to PD [41]. The leucine rich repeat kinase 2 (LRRK2) mutation have some connection with RBD and PD [2].

\section{RBD and Parkinson's disease}

PD is a common and complex neurodegenerative disease in the elderly and clinically featured by motor symptoms, such as bradykinesia, muscular rigidity, rest tremor, and postural and gait impairment, and non-motor symptoms, such as olfactory dysfunction, cognitive impairment, psychiatric symptoms, sleep disorders, autonomic dysfunction, pain, and fatigue [42]. The pathological changes of PD are mainly the loss of dopaminergic neurons and other pigment-containing neurons, especially in the substantia nigra pars compacta ( $\mathrm{SNpc}$ ), and the appearance of eosinophilic inclusion bodies - Lewy bodies, in the cytoplasm of residual neurons. Sleep disorders are very common in PD with an incidence of about $60-70 \%$ [43], and may have a significant negative impact on the quality of life of these patients [44]. Sleep disturbance in PD includes difficulties in falling or staying asleep, sleep fragmentation and daytime sleepiness with involuntarily daytime naps and RBD [45]. In addition, patients with PD are often affected by primary sleep disorders - especially restless legs syndrome (RLS) or obstructive sleep apnea [44].

Many recent studies have shown that RBD in PD marks a subtype of disease characterized by increased risk of cognitive dysfunction and dementia, more autonomic dysfunction, and more multiple gait dysfunctions [46, 47]. Recent studies have shown that RBD is associated with cholinergic denervation [48]. There may be a common cholinergic mechanism between RBD and subsequent increases in the risk of dementia in PD [49]. A meta- analysis has summarized that the prevalence of RBD in $\mathrm{PD}$ is about $42.3 \%$ [50], and more than $70 \%$ of PD patients were male and suffered from dementia in the RBD group [51]. In the semiquantitative analysis of synaptic nucleoprotein deposition, PD patients with RBD usually exhibit more advanced deposition in each brain region examined [51]. The burden of cerebrovascular disease in patients with PD combined with RBD is slightly higher than that in patients without RBD [51]. PD patients with RBD usually have brain structural network alterations on MRI, showing significant enhanced nodal properties in limbic system, frontal-temporal regions, and occipital regions and decreased nodal properties mainly in cerebellum when compared with PD patients without RBD [52].

Pharmacological treatment is not always deemed necessary in RBD. This requires the joint efforts of the patients and co-sleepers. According to Schenk's pioneering observations, clonazepam is the first line-drug to improve the symptom for the majority of patients [53]. Given the long half-life of the drug, careful monitoring must be performed, especially for patients with advanced disease and a higher risk of falls and cognitive impairment [53]. Melatonin has been proposed as second useful agent with fewer side effects [54]. Pramipexole may also be considered, although some studies yield contradictory results [55]. On the other hand, medications used to treat $\mathrm{PD}$, such as monoamine oxidase inhibitors, have been reported to induce RBD symptoms [56].

\section{RBD and Parkinsonism plus syndrome}

Parkinsonism plus syndrome is a group of neurodegenerative diseases featuring the classical features of PD (tremor, rigidity, akinesia/bradykinesia, and postural instability) with additional features that distinguish them from PD, including MSA, PSP, and CBD.

MSA is an adult-onset, sporadic neurodegenerative disease with clinical manifestations of combination of parkinsonian features, cerebellar ataxia, autonomic failure, urogenital dysfunction, and corticospinal disorders [57]. The etiology of MSA is still unclear and most of the current studies suggest that the disease may be caused by a disturbance of synucleinopathy [58]. Sleep disorders are common clinical manifestations of MSA, including reduced and fragmented sleep, excessive daytime sleepiness, RBD, and sleep disordered breathing. Among them, RBD is the most common, affecting 68.8$100 \%$ of MSA patients [33, 59]. RBD may be the first symptom of MSA, ahead of other clinical manifestations for many years [60, 61]. Sleep disorders occur in both subtypes of MSA, MSA with predominant parkinsonism (MSA-P) and MSA with predominant cerebellar ataxia (MSA-C) [62]. Patients with MSA have a significantly 
high rate of RSWA [62]. Due to the progressive deterioration of the neuronal structure in the brainstem, RBD symptoms in MSA patients may disappear as their neurological disease worsens. In addition, degeneration of brainstem nuclei that control REM sleep in patients with advanced MSA may mask RBD symptoms [33]. Concerning the treatment of RBD, it is roughly the same as the treatment of RBD in PD, mainly including the safety management of patients and bed partners and drug treatments [4].

PSP is a progressive tauopathy characterized by supranuclear ophthalmoplegia, pseudobulbar palsy, dysarthria, axial rigidity, frontal lobe dysfunction, and dementia [63]. The typical pathology includes neuronal loss, gliosis and MAPT-positive inclusions in neurons and glial cells, primarily in basal ganglia, brainstem and cerebellum [64]. A study find that RBD and RSWA are presented in 13\% PSP patients [13]. The severe loss of cholinergic neurons in the pedunculopontine tegmentum may contribute to the absence of normal atonia in REM sleep [13]. RBD can be pharmacologically treated with drugs such as clonazepam or melatonin at night [53]. Recently, a study has reported the beneficial effects of combining the dopamine agonist pramipexole with clonazepam [65]. On the other hand, taking paroxetine, fluoxetine, imipramine, venlafaxine, mirtazapine, and $\beta$ blockers can aggravate $\operatorname{RBD}[53,62,66]$.

$\mathrm{CBD}$ is a rare and progressive neurodegenerative disorder. It can present a variety of phenotypes, none of which is specific enough to lead to a definitive diagnosis. One of typical clinical manifestations is corticobasal syndrome, which is usually characterized by asymmetric parkinsonism, a random combination of ideomotor apraxia, rigidity, myoclonus, and dystonia [67]. The ultimate cause of CBD is unclear. However, abnormal accumulations of the MAPT are found in both neurons and glia, supporting the role of tau pathology in CBD [67]. The literatures on RBD in the CBD are mainly limited to case reports and this may be a result of rarity of CBD. 2 female CBD cases were reported with RBD by PSG recording $[38,68]$. Degenerative process in cortical and subcortical structures and in the nuclei of the brainstem and pedunculopontine pathways might play a role in the pathogenesis of RBD in CBD [38].

\section{RBD and Alzheimer's disease}

$\mathrm{AD}$ is the most common cause of dementia in the elderly and characterized by the loss of cognitive function and behavioral disorders. The pathological hallmarks of $A D$ are senile plaques deposited by amloid $\beta(\mathrm{A} \beta)$, neurofibrillary tangles (NFTs) formed by abnormal phosphorylated tau (p-tau) and neuronal loss. It is reported that $24.5-40 \% \mathrm{AD}$ patients have sleep disorders, mainly manifesting as frequent awakenings at night, agitated behavior in the evening and excessive sleeping in the daytime [69]. Sleep disturbance caused by RBD might contribute to $A \beta$ and tau pathologies and aggravate cognitive impairment [70].

Although it is generally considered that RBD is a strong predictor of neurodegeneration in particular synucleinopathies, studies have indicated RBD in a minority of $\mathrm{AD}$ patients [12]. A study of probable $\mathrm{AD}$ patients has showed that 4 out of 15 patients with $A D$ presented RSWA, and one of these patients had all the polysomnographic features of RBD [71]. Another study of 105 probable AD patients has reported that 5 patients with $\mathrm{AD}$ exhibited RBD [12]. AD patients with RBD usually show increases in both tonic and phasic electromyography activity during REM sleep, but no sleep architecture differences between AD patients with and without RBD [12]. A MRI study has showed more specialized atrophic patterns distributed in the posterior and inferior parts of the brain, including the bilateral temporal and occipital cortices in AD patients with RBD compared to non-RBD AD patients [72]. On the other hand, a longitudinal study with about 4.2-year follow-up has demonstrated that 18 out of 84 patients with iRBD developed neurodegenerative diseases, and 3 of these patients were diagnosed with AD [73]. Another study of 179 iRBD patients with mean follow-up of 5.8 years has found that $50(27.9 \%)$ iRBD patients turned out to have neurodegenerative diseases, 14 of these patients were diagnosed with probable AD [74]. Because the neurotransmitter acetylcholine is related to the induction of atonia during REM sleep, it is suggested that the incidence of RBD is associated with decreased acetylcholine level in AD patients [12].

For the treatment of RBD, modifying the sleep environment is recommended to avoid sleep-related injury. Clonazepam is suggested for the treatment of RBD but should be used with caution in patients with dementia, gait disorders, or concomitant obstructive sleep apnea (OSA) [53]. Melatonin has been reported as an alternative treatment in $\mathrm{AD}$ patient with $\mathrm{RBD}$ and sleep disordered breathing [75]. And medication for the treatment of dementia, such as rivastigmine, might induce RBD in AD patients [76].

\section{RBD and other dementias}

Apart from $\mathrm{AD}$, other diseases with different pathogeneses also cause cognitive impairment and lead to dementia, including DLB, FTD, and vascular dementia $(\mathrm{VaD})$.

DLB is characterized by cognitive impairment, Parkinson's syndrome and visual hallucinations [77]. It is pathologically featured by intracytoplasmic inclusions 
called Lewy bodies, which consist of filamentous protein granules composed of $\alpha$-syn and ubiquitin found in brainstem nuclei and the neocortex [77]. DLB also demonstrates some pathological features of $\mathrm{AD}$, including $\mathrm{A} \beta$ deposits and NFTs [77]. An autopsy study has reported that striatal $A \beta$-immunoreactive plaques are found in DLB and positively correlated with the severity of dementia [78]. A $\beta$ deposition usually predicts DLB cases with high specificity and sensitivity [79]. Another study has found that $71 \%$ of the DLB patients are amyloid positive and $17 \%$ of the DLB patients are also positive for p-tau [80]. These results imply that AD-like pathologies might play a crucial role in pathogenesis of DLB [78]. Patients with DLB usually have a high prevalence of RBD around 46.7-47.6\% [81, 82]. A PSG study has reported that $83 \%$ DLB patients showed RSWA with or without dream enactment during PSG [83]. RBD is considered as a strong predictor of neurodegeneration which precede for many years the onset of synucleinopathy [84]. Longitudinal study has reported that RBD might predict the new development of hallucinations and cognitive fluctuations while RSWA might predict development of dementia in PD patients [85]. DLB patients with RBD also exhibits brain metabolic differences, presenting severe metabolic decrease in the dorsolateral and medial frontal regions, left precuneus, bilateral superior parietal lobule and rolandic operculum, and amygdala [80]. A MRI study has reported that the presence of RBD is associated with a higher likelihood of DLB and less severe AD-related pathology in the medial temporal lobes, whereas absence of RBD is characterized by AD-like atrophy patterns on MRI and increased p-tau burden, indicating that RBD is specifically linked to synucleinopathy [82]. Melatonin, clonazepam, and ramelteon can be used to treat RBD in DLB [86].

FTD is characterized by deterioration in behavior and personality, language disturbances, or alterations in muscle or motor functions, resulting from progressive neurodegeneration of the frontal and temporal lobes [87]. The pathogenesis of FTD is heterogeneous due to the different molecular subtypes of clinical FTD [88]. Most frontotemporal degenerations are associated with the MAPT, TAR DNA-binding protein with molecular weight $43 \mathrm{kDa}$ (TDP-43), and fused-in-sarcoma (FUS) protein [87]. A few cases of frontotemporal lobar degeneration have ubiquitin-only or p62-only positive inclusions, or no inclusions at all [87]. It is reported that 76\% patients with FTD exhibit sleep disturbance, increased nocturnal activity and decreased morning activity [81]. RBD is rarely reported in FTD and may be misdiagnosed due to the high rate of nocturnal activity and altered sleep-wake cycle in FTD patients [89]. A case report has documented PSG-confirmed RBD in an FTD patient, and the symptoms of RBD include unpleasant dreams, vocalization, waving hands to fight and kicking legs, preceed emotional and behavioral changes of FTD by 3 years [90]. Olanzapine and clonazepam are often prescribed to treat FTD with RBD [90]. Since chromosome 9 open reading frame 72 (C9ORF72) repeat expansion is reported presented in RBD [91], which has also been reported to occur in $25.1 \%$ of FTD patients, C9ORF72 expansion might be responsible for the evolution of RBD in FTD [89].

$\mathrm{VaD}$ is a cognitive impairment caused by vascular conditions which reduce the blood flow to the brain. The symptoms of $\mathrm{VaD}$ depend on the brain areas affected by the hypoperfusion or hemodynamic abnormality [92]. Previous study has indicated that sleep disturbance, including insomnia, sleep-disordered breathing, RBD, restless legs syndrome, excessive daytime sleepiness occurred in $81.4 \%$ of the patients with $\mathrm{VaD}$, and $25.6 \%$ $\mathrm{VaD}$ patients have RBD [81]. More recent study has reported a higher prevalence $(72.6 \%)$ of $\mathrm{RBD}$ in $\mathrm{VaD}$ patients [93]. Since lesions in the LC or SLD cause RSWA, hypoperfusion or hemodynamic abnormalities in these brain areas that regulate REM sleep may contribute to the pathogenesis of RBD in $\mathrm{VaD}$ [4].

\section{RBD and Huntington's disease}

HD is a progressive, neurodegenerative disorder that usually causes movement, cognitive and psychiatric problems with a wide spectrum of signs and symptoms [94]. It is a monogenic autosomal dominant disorder by a CAG triplet repeat expansion in huntingtin (HTT) gene, which encodes an expanded polyglutamine stretch in the HTT protein [94]. Evidence suggests that HD arises predominantly from gain of a toxic function from an abnormal conformation of mutant HTT [94]. Sleep disorders in HD include frequent insomnia, earlier sleep onset, lower sleep efficiency, increased stage 1 sleep, delayed and shortened REM sleep, and increased periodic leg movements [14]. Circadian rhythm disruption is also a prominent feature of sleep disorders in HD [95]. A study has reported that 3 out of $25 \mathrm{HD}$ patients have RBD, manifesting as complex movements of the lips, head, trunk, and right hand and arm during REM sleep [14]. However, in another polysomnographic study, no episode of RBD is observed in $30 \mathrm{HD}$ patients [96].

\section{RBD and amyotrophic lateral sclerosis}

ALS is an adult-onset progressive neurodegenerative disease, characterized by rapidly progressive loss of cortex, spinal cord, bulbar movement neurons, manifesting with paralysis of the striatum skeletal muscle, bulbar muscle, dysphagia, dysarthria, and respiratory dysfunction [97]. ALS patients usually die in 1-5 years 
resulting from respiratory failure [97]. ALS patients often suffer from sleep disorders including insomnia, fragmented sleep, and increased periodic limb movement disorder [15]. Previous studies have focused on sleep disorders caused by chronic respiratory muscle paralysis and hypoventilation in ALS patients [98, 99]. RBD has also been reported in ALS patients. A study of 41 ALS patients and 26 healthy participants has found that 2 ALS patients had RBD, 2 had RSWA, and the control group had normal REM sleep [15]. Mechanism of RBD in ALS patients is uncertain. The early death caused by respiration in ALS patients affects the observation of RBD in later stage [97]. Moreover, the absence of ALS motor neurons also prevents the motor behaviors during sleep. During
REM, the tone of the chin muscles changed in ALS [97]. However, it is uncertain if the alteration of the muscle tone during REM sleep results from the loss of innervation because of the degeneration of lower motor neurons, or if it can parallel spasticity and hyperreflexia associated with the degeneration of upper motor neurons [97]. The most common forms of ALS and FTD are caused by GGGGCC hexanucleotide repeat expansions in the first intron of C9ORF72 [100]. A study of 344 patients with RBD has found that 2 RBD patients have the same C9ORF72 associated-risk haplotype identified in ALS and FTD, suggesting that this rare expansion might be associated with the pathogenesis of RBD [91].

Table 1. Summarized prevalence, gender difference and underlying mechanisms of RBD in neurodegenerative diseases.

\begin{tabular}{|c|c|c|c|}
\hline Disease & Prevalence & Gender difference & Possible mechanisms of pathogenesis of RBD \\
\hline PD & $42.3 \%[50]$ & Male>female [51] & $\begin{array}{l}\alpha \text {-syn pathology affects the circuit that regulates REM sleep, } \\
\text { associated with GBA [39], SCARB2 [40], MAPT [40], } \\
\text { USP25[40], PINK1 [41], LRRK2 [2] mutations }\end{array}$ \\
\hline MSA & $88 \%[33]$ & Female >male [59] & $\begin{array}{l}\alpha \text {-syn pathology affects the circuit that regulates REM sleep, } \\
\text { depletion of cholinergic neurons in the PPN/LDTN } \\
\text { complex, periaqueductal grey matter, and LC [33] }\end{array}$ \\
\hline PSP & $13 \%[13]$ & - & $\begin{array}{l}\text { Loss of cholinergic neurons in the pedunculopontine } \\
\text { tegmentum [13] }\end{array}$ \\
\hline CBD & Case reports $[38,68]$ & 2 female patients $[38,68]$ & $\begin{array}{l}\text { Degenerative process in cortical and subcortical structures } \\
\text { and in the nuclei of the brain stem and pedunculopontine } \\
\text { pathways [38]. }\end{array}$ \\
\hline $\mathrm{AD}$ & $4.8-26.7 \%[12,71]$ & Male>female [12] & $\begin{array}{l}\text { An imbalance of neurotransmitter acetylcholine [12], } \\
\text { neuronal loss in LC [12] }\end{array}$ \\
\hline DLB & $46.7-83 \%[81-83]$ & Male>female [82] & $\begin{array}{l}\alpha \text {-syn pathology affects the circuit that regulates REM sleep } \\
{[4]}\end{array}$ \\
\hline FTD & Rare (only case report) $[90]$ & 1 male patient $[90]$ & Associated with C9ORF72 repeat expansion [89] \\
\hline $\mathrm{VaD}$ & $25.6 \%-72.6 \%[81,93]$ & 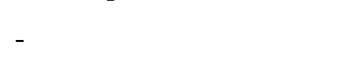 & $\begin{array}{l}\text { Hypoperfusion or hemodynamic abnormalities affect the } \\
\text { brain areas that regulate REM sleep [4] }\end{array}$ \\
\hline HD & $12 \%$ or lower $[14,96]$ & Female >male [14] & Associated with mutant huntingtin [14] \\
\hline ALS & $4.9 \%[15]$ & 2 male patients $[15]$ & $\begin{array}{l}\text { Neurodegeneration of nuclei in REM-associated pathways } \\
\text { in the brainstem and the dysfunction of dopaminergic system } \\
\text { in substantia nigra striatum may be the main } \\
\text { pathophysiological culprit in the development of RBD [15], } \\
\text { associated with C9ORF72 repeat expansion }[91,100]\end{array}$ \\
\hline CJD & $7.1 \%[101]$ & 2 male patients $[101]$ & Associated with corticothalamic degeneration [102] \\
\hline
\end{tabular}

\section{RBD and Creutzfeldt-Jakob disease}

Creutzfeldt-Jakob disease (CJD) is a rapid progressive, fatal neurodegenerative diseases. The main pathogenesis is the accumulation of misfolded prion protein, $\mathrm{PrPSc}$ [101]. Sleep disorders are common in CJD. Early treatment and prevention of sleep disorders can interfere with the treatment of CJD. Although sleep symptoms are not considered to be the main symptoms of CJD, sleepwake disorder is an important source of morbidity and reduces the quality of life in these patients [101]. A crosssectional cohort study has retrospectively analyzed 28 patients diagnosed CJD and found that 3 patients had RSWA, and 2 had RBD [101]. This study suggests that $\mathrm{RBD}$ is as common in CJD patients as it is in patients with synucleinopathies. RBD in CJD might result from 
corticothalamic degeneration [102]. Due to methodology and limited sample size, larger studies involving multiple centers are needed.

\section{Summary}

RBD is generally considered to be associated with synucleinopathy, but it is also reported in other neurodegenerative diseases including AD, FTD, HD, ALS, etc, which are not characterized by $\alpha$-syn pathology. The summarized prevalence, gender difference and underlying mechanisms of RBD in neurodegenerative diseases is listed in Table 1. RBD may precede years before the onset of the symptoms of synucleinopathy and predict neurodegeneration and cognitive impairment. The underlying mechanisms of the pathogenesis of RBD is not clear. Since the neural circuit in caudal brainstem that regulates REM sleep is where $\alpha$-syn pathology may begin in synucleinopathy, RBD may result from the degeneration of circuits that regulate the REM sleep, suggesting that the $\alpha$-syn pathological process may start years before the motor or cognitive symptoms of these diseases. Sleep changes might be a very early sign of neurodegeneration in synucleinopathy. On the other hand, $\mathrm{RBD}$ in PD is associated with a more impaired cognitive profile, suggesting more severe and widespread neurodegeneration [103], implying sleep disturbance might also be an assessment of cognitive deficits in synucleinopathies. In non-synucleinopahties, RBD may be associated with co-existing $\alpha$-syn pathology [104] or other pathological changes affecting the pathways in the brainstem controlling REM [13, 15, 38]. Thus, the site of the lesion is more critical in the pathogenesis of RBD, rather than the type of pathological changes. For the treatment of RBD, clonazepam, melatonin, and ramelteon can be applied. Medications used to treat the neurodegenerative diseases, such as monoamine oxidase inhibitors, antidepressant, might induce or deteriorate the symptoms of RBD.

\section{Acknowledgements}

This work was supported in part by funding from the National Natural Science Foundation of China (NSFC 81430021 and 81771521) and the Key Field Research and Development Program of Guangdong Province (2018B030337001). All the informations prepared for this review paper came from PubMed with the relevant publications until January 2019, including original clinical and basic research articles, meta and metaregression analysis, and prospective/retro-spective reviews.

\section{References}

[1] Schenck CH, Mahowald MW (2002). REM sleep behavior disorder: clinical, developmental, and neuroscience perspectives 16 years after its formal identification in SLEEP. Sleep, 25:120-138.

[2] St Louis EK, Boeve BF (2017). REM Sleep Behavior Disorder: Diagnosis, Clinical Implications, and Future Directions. Mayo Clin Proc, 92:1723-1736.

[3] St Louis EK, Boeve AR, Boeve BF (2017). REM Sleep Behavior Disorder in Parkinson's Disease and Other Synucleinopathies. Mov Disord, 32:645-658.

[4] McCarter SJ, St Louis EK, Boeve BF (2012). REM sleep behavior disorder and REM sleep without atonia as an early manifestation of degenerative neurological disease. Curr Neurol Neurosci Rep, 12:182-192.

[5] Hogl B, Stefani A, Videnovic A (2018). Idiopathic REM sleep behaviour disorder and neurodegeneration - an update. Nat Rev Neurol, 14:40-55.

[6] Li M, Wang L, Liu JH, Zhan SQ (2018). Relationships between Rapid Eye Movement Sleep Behavior Disorder and Neurodegenerative Diseases: Clinical Assessments, Biomarkers, and Treatment. Chin Med J (Engl), 131:966-973.

[7] Reichmann H (2017). Premotor Diagnosis of Parkinson's Disease. Neurosci Bull, 33:526-534.

[8] Mollenhauer B, Trautmann E, Sixel-Doring F, Wicke T, Ebentheuer J, Schaumburg M, et al. (2013). Nonmotor and diagnostic findings in subjects with de novo Parkinson disease of the DeNoPa cohort. Neurology, 81:1226-1234.

[9] Boot BP (2015). Comprehensive treatment of dementia with Lewy bodies. Alzheimers Res Ther, 7:45.

[10] Arnaldi D, Antelmi E, St Louis EK, Postuma RB, Arnulf I (2017). Idiopathic REM sleep behavior disorder and neurodegenerative risk: To tell or not to tell to the patient? How to minimize the risk? Sleep Med Rev, 36:82-95.

[11] Sabater L, Gaig C, Gelpi E, Bataller L, Lewerenz J, Torres-Vega E, et al. (2014). A novel non-rapid-eye movement and rapid-eye-movement parasomnia with sleep breathing disorder associated with antibodies to IgLON5: a case series, characterisation of the antigen, and post-mortem study. Lancet Neurol, 13:575-586.

[12] Wang P, Wing YK, Xing J, Liu Y, Zhou B, Zhang Z, et al. (2016). Rapid eye movement sleep behavior disorder in patients with probable Alzheimer's disease. Aging Clin Exp Res, 28:951-957.

[13] Arnulf I, Merino-Andreu M, Bloch F, Konofal E, Vidailhet M, Cochen V, et al. (2005). REM sleep behavior disorder and REM sleep without atonia in patients with progressive supranuclear palsy. Sleep, 28:349-354.

[14] Arnulf I, Nielsen J, Lohmann E, Schiefer J, Wild E, Jennum P, et al. (2008). Rapid eye movement sleep disturbances in Huntington disease. Arch Neurol, 65:482-488.

[15] Lo Coco D, Puligheddu M, Mattaliano P, Congiu P, Borghero G, Fantini ML, et al. (2017). REM sleep behavior disorder and periodic leg movements during 
sleep in ALS. Acta Neurol Scand, 135:219-224.

[16] Rodriguez CL, Jaimchariyatam N, Budur K (2017). Rapid Eye Movement Sleep Behavior Disorder: A Review of the Literature and Update on Current Concepts. Chest, 152:650-662.

[17] Borek LL, Kohn R, Friedman JH (2007). Phenomenology of dreams in Parkinson's disease. Mov Disord, 22:198-202.

[18] Bassetti CL, Bargiotas P (2018). REM Sleep Behavior Disorder. Front Neurol Neurosci, 41:104-116.

[19] Howell MJ, Schenck CH (2015). Rapid Eye Movement Sleep Behavior Disorder and Neurodegenerative Disease. JAMA Neurol, 72:707-712.

[20] Chiaro G, Calandra-Buonaura G, Cecere A, Mignani F, Sambati L, Loddo G, et al. (2017). REM sleep behavior disorder, autonomic dysfunction and synuclein-related neurodegeneration: where do we stand? Clin Auton Res:1-15.

[21] Boeve BF (2010). REM sleep behavior disorder: Updated review of the core features, the REM sleep behavior disorder-neurodegenerative disease association, evolving concepts, controversies, and future directions. Ann N Y Acad Sci, 1184:15-54.

[22] Jin H, Zhang JR, Shen Y, Liu CF (2017). Clinical Significance of REM Sleep Behavior Disorders and Other Non-motor Symptoms of Parkinsonism. Neurosci Bull, 33:576-584.

[23] Unger MM, Belke M, Menzler K, Heverhagen JT, Keil B, Stiasny-Kolster K, et al. (2010). Diffusion tensor imaging in idiopathic REM sleep behavior disorder reveals microstructural changes in the brainstem, substantia nigra, olfactory region, and other brain regions. Sleep, 33:767-773.

[24] Scherfler C, Frauscher B, Schocke M, Iranzo A, Gschliesser V, Seppi K, et al. (2011). White and gray matter abnormalities in idiopathic rapid eye movement sleep behavior disorder: a diffusion-tensor imaging and voxel-based morphometry study. Ann Neurol, 69:400407.

[25] Pyatigorskaya N, Gaurav R, Arnaldi D, Leu-Semenescu S, Yahia-Cherif L, Valabregue R, et al. (2017). Magnetic Resonance Imaging Biomarkers to Assess Substantia Nigra Damage in Idiopathic Rapid Eye Movement Sleep Behavior Disorder. Sleep, 40.

[26] Rahayel S, Montplaisir J, Monchi O, Bedetti C, Postuma RB, Brambati S, et al. (2015). Patterns of cortical thinning in idiopathic rapid eye movement sleep behavior disorder. Mov Disord, 30:680-687.

[27] Bae YJ, Kim JM, Kim KJ, Kim E, Park HS, Kang SY, et al. (2018). Loss of Substantia Nigra Hyperintensity at 3.0-T MR Imaging in Idiopathic REM Sleep Behavior Disorder: Comparison with (123)I-FP-CIT SPECT. Radiology, 287:285-293.

[28] Skorvanek M, Feketeova E, Kurtis MM, Rusz J, Sonka K (2018). Accuracy of Rating Scales and Clinical Measures for Screening of Rapid Eye Movement Sleep Behavior Disorder and for Predicting Conversion to Parkinson's Disease and Other Synucleinopathies. Front Neurol, 9:376.

[29] Fraigne JJ, Torontali ZA, Snow MB, Peever JH (2015).
REM Sleep at its Core - Circuits, Neurotransmitters, and Pathophysiology. Front Neurol, 6:123.

[30] McKenna D, Peever J (2017). Degeneration of rapid eye movement sleep circuitry underlies rapid eye movement sleep behavior disorder. Mov Disord, 32:636-644.

[31] Dauvilliers Y, Schenck CH, Postuma RB, Iranzo A, Luppi PH, Plazzi G, et al. (2018). REM sleep behaviour disorder. Nat Rev Dis Primers, 4:19.

[32] Knudsen S, Gammeltoft S, Jennum PJ (2010). Rapid eye movement sleep behaviour disorder in patients with narcolepsy is associated with hypocretin-1 deficiency. Brain, 133:568-579.

[33] Palma JA, Fernandez-Cordon C, Coon EA, Low PA, Miglis MG, Jaradeh S, et al. (2015). Prevalence of REM sleep behavior disorder in multiple system atrophy: a multicenter study and meta-analysis. Clin Auton Res, 25:69-75.

[34] Iranzo A, Tolosa E, Gelpi E, Molinuevo JL, Valldeoriola F, Serradell M, et al. (2013). Neurodegenerative disease status and post-mortem pathology in idiopathic rapideye-movement sleep behaviour disorder: an observational cohort study. Lancet Neurol, 12:443-453.

[35] Peever J, Fuller PM (2016). Neuroscience: A Distributed Neural Network Controls REM Sleep. Curr Biol, 26:R34-35.

[36] Braak H, Del Tredici K, Rub U, de Vos RA, Jansen Steur EN, Braak E (2003). Staging of brain pathology related to sporadic Parkinson's disease. Neurobiol Aging, 24:197-211.

[37] Braak H, Rub U, Gai WP, Del Tredici K (2003). Idiopathic Parkinson's disease: possible routes by which vulnerable neuronal types may be subject to neuroinvasion by an unknown pathogen. J Neural Transm (Vienna), 110:517-536.

[38] Wetter TC, Brunner H, Collado-Seidel V, Trenkwalder C, Winkelmann J (2002). Sleep and periodic limb movements in corticobasal degeneration. Sleep Med, 3:33-36.

[39] Gan-Or Z, Mirelman A, Postuma RB, Arnulf I, Bar-Shira A, Dauvilliers Y, et al. (2015). GBA mutations are associated with Rapid Eye Movement Sleep Behavior Disorder. Ann Clin Transl Neurol, 2:941-945.

[40] Gan-Or Z, Girard SL, Noreau A, Leblond CS, Gagnon JF, Arnulf I, et al. (2015). Parkinson's Disease Genetic Loci in Rapid Eye Movement Sleep Behavior Disorder. J Mol Neurosci, 56:617-622.

[41] Puschmann A, Fiesel FC, Caulfield TR, Hudec R, Ando M, Truban D, et al. (2017). Heterozygous PINK1 p.G411S increases risk of Parkinson's disease via a dominant-negative mechanism. Brain, 140:98-117.

[42] Kalia LV, Lang AE (2015). Parkinson's disease. Lancet, 386:896-912.

[43] Ma JF, Hou MM, Tang HD, Gao X, Liang L, Zhu LF, et al. (2016). REM sleep behavior disorder was associated with Parkinson's disease: a community-based study. BMC Neurol, 16:123.

[44] Schulte EC, Winkelmann J (2011). When Parkinson's disease patients go to sleep: specific sleep disturbances related to Parkinson's disease. J Neurol, 258:S328-335.

[45] Schrempf W, Brandt MD, Storch A, Reichmann H (2014). 
Sleep disorders in Parkinson's disease. J Parkinsons Dis, 4:211-221.

[46] Fereshtehnejad SM, Romenets SR, Anang JB, Latreille V, Gagnon JF, Postuma RB (2015). New Clinical Subtypes of Parkinson Disease and Their Longitudinal Progression: A Prospective Cohort Comparison With Other Phenotypes. JAMA Neurol, 72:863-873.

[47] Romenets SR, Gagnon JF, Latreille V, Panniset M, Chouinard S, Montplaisir J, et al. (2012). Rapid eye movement sleep behavior disorder and subtypes of Parkinson's disease. Mov Disord, 27:996-1003.

[48] Muller ML, Bohnen NI (2013). Cholinergic dysfunction in Parkinson's disease. Curr Neurol Neurosci Rep, 13:377.

[49] Marion MH, Qurashi M, Marshall G, Foster O (2008). Is REM sleep behaviour disorder (RBD) a risk factor of dementia in idiopathic Parkinson's disease? J Neurol, 255:192-196.

[50] Zhang X, Sun X, Wang J, Tang L, Xie A (2017). Prevalence of rapid eye movement sleep behavior disorder (RBD) in Parkinson's disease: a meta and metaregression analysis. Neurol Sci, 38:163-170.

[51] Postuma RB, Adler CH, Dugger BN, Hentz JG, Shill HA, Driver-Dunckley E, et al. (2015). REM sleep behavior disorder and neuropathology in Parkinson's disease. Mov Disord, 30:1413-1417.

[52] Guo T, Guan X, Zeng Q, Xuan M, Gu Q, Huang P, et al. (2018). Alterations of Brain Structural Network in Parkinson's Disease With and Without Rapid Eye Movement Sleep Behavior Disorder. Front Neurol, 9:334.

[53] Aurora RN, Zak RS, Maganti RK, Auerbach SH, Casey KR, Chowdhuri S, et al. (2010). Best practice guide for the treatment of REM sleep behavior disorder (RBD). J Clin Sleep Med, 6:85-95.

[54] Kunz D, Mahlberg R (2010). A two-part, double-blind, placebo-controlled trial of exogenous melatonin in REM sleep behaviour disorder. J Sleep Res, 19:591-596.

[55] Chaudhuri KR, Schapira AH (2009). Non-motor symptoms of Parkinson's disease: dopaminergic pathophysiology and treatment. Lancet Neurol, 8:464474.

[56] Gagnon JF, Postuma RB, Montplaisir J (2006). Update on the pharmacology of REM sleep behavior disorder. Neurology, 67:742-747.

[57] Ubhi K, Low P, Masliah E (2011). Multiple system atrophy: a clinical and neuropathological perspective. Trends Neurosci, 34:581-590.

[58] Fellner L, Jellinger KA, Wenning GK, Stefanova N (2011). Glial dysfunction in the pathogenesis of alphasynucleinopathies: emerging concepts. Acta Neuropathol, 121:675-693.

[59] Nomura T, Inoue Y, Hogl B, Uemura Y, Yasui K, Sasai T, et al. (2011). Comparison of the clinical features of rapid eye movement sleep behavior disorder in patients with Parkinson's disease and multiple system atrophy. Psychiatry Clin Neurosci, 65:264-271.

[60] Zesiewicz TA, Sullivan KL, Gooch CL (2008). Red flags to spot the parkinsonian variant of multiple system atrophy. Nat Clin Pract Neurol, 4:596-597.

[61] Postuma RB, Gagnon JF, Vendette M, Fantini ML,
Massicotte-Marquez J, Montplaisir J (2009). Quantifying the risk of neurodegenerative disease in idiopathic REM sleep behavior disorder. Neurology, 72:1296-1300.

[62] Boeve BF, Silber MH, Saper CB, Ferman TJ, Dickson DW, Parisi JE, et al. (2007). Pathophysiology of REM sleep behaviour disorder and relevance to neurodegenerative disease. Brain, 130:2770-2788.

[63] Long L, Cai XD, Wei XB, Liao JC, Xu YQ, Gao HM, et al. (2015). Progressive supranuclear palsy: what do we know about it? Curr Med Chem, 22:1182-1193.

[64] Schrag A, Selai C, Davis J, Lees AJ, Jahanshahi M, Quinn N (2003). Health-related quality of life in patients with progressive supranuclear palsy. Mov Disord, 18:1464-1469.

[65] Sasai T, Matsuura M, Inoue Y (2013). Factors associated with the effect of pramipexole on symptoms of idiopathic REM sleep behavior disorder. Parkinsonism Relat Disord, 19:153-157.

[66] Knie B, Mitra MT, Logishetty K, Chaudhuri KR (2011). Excessive daytime sleepiness in patients with Parkinson's disease. CNS Drugs, 25:203-212.

[67] Boeve BF (2011). The multiple phenotypes of corticobasal syndrome and corticobasal degeneration: implications for further study. J Mol Neurosci, 45:350353.

[68] Kimura K, Tachibana N, Aso T, Kimura J, Shibasaki H (1997). Subclinical REM sleep behavior disorder in a patient with corticobasal degeneration. Sleep, 20:891894.

[69] Zhang F, Zhong R, Li S, Chang RC, Le W (2017). The missing link between sleep disorders and age-related dementia: recent evidence and plausible mechanisms. J Neural Transm (Vienna), 124:559-568.

[70] Qiu H, Zhong R, Liu H, Zhang F, Li S, Le W (2016). Chronic Sleep Deprivation Exacerbates LearningMemory Disability and Alzheimer's Disease-Like Pathologies in AbetaPP(swe)/PS1(DeltaE9) Mice. J Alzheimers Dis, 50:669-685.

[71] Gagnon JF, Petit D, Fantini ML, Rompre S, Gauthier S, Panisset M, et al. (2006). REM sleep behavior disorder and REM sleep without atonia in probable Alzheimer disease. Sleep, 29:1321-1325.

[72] Kim HJ, Im HK, Kim J, Han JY, de Leon M, Deshpande A, et al. (2016). Brain Atrophy of Secondary REM-Sleep Behavior Disorder in Neurodegenerative Disease. J Alzheimers Dis, 52:1101-1109.

[73] Youn S, Kim T, Yoon IY, Jeong J, Kim HY, Han JW, et al. (2016). Progression of cognitive impairments in idiopathic REM sleep behaviour disorder. J Neurol Neurosurg Psychiatry, 87:890-896.

[74] Zhou J, Zhang J, Lam SP, Chan JW, Mok V, Chan A, et al. (2017). Excessive Daytime Sleepiness Predicts Neurodegeneration in Idiopathic REM Sleep Behavior Disorder. Sleep, 40:zsx041.

[75] Anderson KN, Jamieson S, Graham AJ, Shneerson JM (2008). REM sleep behaviour disorder treated with melatonin in a patient with Alzheimer's disease. Clin Neurol Neurosurg, 110:492-495.

[76] Yeh SB, Yeh PY, Schenck CH (2010). Rivastigmine- 
induced REM sleep behavior disorder (RBD) in a 88year-old man with Alzheimer's disease. J Clin Sleep Med, 6:192-195.

[77] Mayo MC, Bordelon Y (2014). Dementia with Lewy bodies. Semin Neurol, 34:182-188.

[78] Halliday GM, Song YJ, Harding AJ (2011). Striatal betaamyloid in dementia with Lewy bodies but not Parkinson's disease. J Neural Transm (Vienna), 118:713719.

[79] Provini F (2013). Agrypnia excitata. Curr Neurol Neurosci Rep, 13:341.

[80] Iaccarino L, Marelli S, Iannaccone S, Magnani G, FeriniStrambi L, Perani D (2016). Severe Brain Metabolic Decreases Associated with REM Sleep Behavior Disorder in Dementia with Lewy Bodies. J Alzheimers Dis, 52:989-997.

[81] Guarnieri B, Adorni F, Musicco M, Appollonio I, Bonanni E, Caffarra P, et al. (2012). Prevalence of sleep disturbances in mild cognitive impairment and dementing disorders: a multicenter Italian clinical crosssectional study on 431 patients. Dement Geriatr Cogn Disord, 33:50-58.

[82] Murray ME, Ferman TJ, Boeve BF, Przybelski SA, Lesnick TG, Liesinger AM, et al. (2013). MRI and pathology of REM sleep behavior disorder in dementia with Lewy bodies. Neurology, 81:1681-1689.

[83] Pao WC, Boeve BF, Ferman TJ, Lin SC, Smith GE, Knopman DS, et al. (2013). Polysomnographic findings in dementia with Lewy bodies. Neurologist, 19:1-6.

[84] Fulda S (2011). Idiopathic REM sleep behavior disorder as a long-term predictor of neurodegenerative disorders. EPMA J, 2:451-458.

[85] Postuma RB, Bertrand JA, Montplaisir J, Desjardins C, Vendette M, Rios Romenets S, et al. (2012). Rapid eye movement sleep behavior disorder and risk of dementia in Parkinson's disease: a prospective study. Mov Disord, 27:720-726.

[86] Tousi B (2017). Diagnosis and Management of Cognitive and Behavioral Changes in Dementia With Lewy Bodies. Curr Treat Options Neurol, 19:42.

[87] Bang J, Spina S, Miller BL (2015). Frontotemporal dementia. Lancet, 386:1672-1682.

[88] Mackenzie IR, Neumann M (2016). Molecular neuropathology of frontotemporal dementia: insights into disease mechanisms from postmortem studies. J Neurochem, 138 Suppl 1:54-70.

[89] McCarter SJ, St Louis EK, Boeve BF (2016). Sleep Disturbances in Frontotemporal Dementia. Curr Neurol Neurosci Rep, 16:85.

[90] Lo Coco D, Cupidi C, Mattaliano A, Baiamonte V, Realmuto S, Cannizzaro E (2012). REM sleep behavior disorder in a patient with frontotemporal dementia. Neurol Sci, 33:371-373.

[91] Meloni M, Farris R, Solla P, Mascia MM, Marrosu F,
Cannas A (2017). C9ORF72 Intermediate Repeat Expansion in a Patient With Psychiatric Disorders and Progressive Cerebellar Ataxia. Neurologist, 22:245-246.

[92] O'Brien JT, Thomas A (2015). Vascular dementia. Lancet, 386:1698-1706.

[93] Pistacchi M, Gioulis M, Contin F, Sanson F, Marsala SZ (2014). Sleep disturbance and cognitive disorder: epidemiological analysis in a cohort of 263 patients. Neurol Sci, 35:1955-1962.

[94] Ross CA, Tabrizi SJ (2011). Huntington's disease: from molecular pathogenesis to clinical treatment. Lancet Neurol, 10:83-98.

[95] Anderson KE, van Duijn E, Craufurd D, Drazinic C, Edmondson M, Goodman N, et al. (2018). Clinical Management of Neuropsychiatric Symptoms of Huntington Disease: Expert-Based Consensus Guidelines on Agitation, Anxiety, Apathy, Psychosis and Sleep Disorders. J Huntingtons Dis, 7:239-250.

[96] Piano C, Losurdo A, Della Marca G, Solito M, CalandraBuonaura G, Provini F, et al. (2015). Polysomnographic Findings and Clinical Correlates in Huntington Disease: A Cross-Sectional Cohort Study. Sleep, 38:1489-1495.

[97] Puligheddu M, Congiu P, Arico D, Rundo F, Borghero G, Marrosu F, et al. (2016). Isolated rapid eye movement sleep without atonia in amyotrophic lateral sclerosis. Sleep Med, 26:16-22.

[98] Yamauchi R, Imai T, Tsuda E, Hozuki T, Yamamoto D, Shimohama S (2014). Respiratory insufficiency with preserved diaphragmatic function in amyotrophic lateral sclerosis. Intern Med, 53:1325-1331.

[99] Ahmed RM, Newcombe RE, Piper AJ, Lewis SJ, Yee BJ, Kiernan MC, et al. (2016). Sleep disorders and respiratory function in amyotrophic lateral sclerosis. Sleep Med Rev, 26:33-42.

[100] Pugliatti M, Parish LD, Cossu P, Leoni S, Ticca A, Saddi $\mathrm{MV}$, et al. (2013). Amyotrophic lateral sclerosis in Sardinia, insular Italy, 1995-2009. J Neurol, 260:572579.

[101] Kang P, de Bruin GS, Wang LH, Ward BA, Ances BM, Lim MM, et al. (2016). Sleep Pathology in CreutzfeldtJakob Disease. J Clin Sleep Med, 12:1033-1039.

[102] Puligheddu M, Congiu P, Laccu I, Figorilli M, Gioi G, Polizzi L, et al. (2017). Overlap Parasomnia Disorder in a case of Creutzfeldt-Jakob Disease. Sleep Med, 36:7577.

[103] Jozwiak N, Postuma RB, Montplaisir J, Latreille V, Panisset M, Chouinard S, et al. (2017). REM Sleep Behavior Disorder and Cognitive Impairment in Parkinson's Disease. Sleep, 40:zsx101.

[104] Korff A, Liu C, Ginghina C, Shi M, Zhang J, Alzheimer's Disease Neuroimaging I (2013). alpha-Synuclein in cerebrospinal fluid of Alzheimer's disease and mild cognitive impairment. J Alzheimers Dis, 36:679-688. 